Charles BONNOT

Université Paris Diderot-Paris 7

CLILLAC -ARP (Centre de Linguistique Inter-langues, de Lexicologie, de Linguistique Anglaise et de Corpus)

\title{
Les premières fois : récits des origines dans les documentaires musicaux
}

\author{
That was my first time in America. Sid \\ [Vicious] would sit next to me, and we'd \\ look out the window. (...) You wouldn't \\ sleep. You wouldn't want to, because it \\ was so first time. \\ Johnny Rotten [Sex Pistols], The Filth \\ and the Fury
}

\section{Introduction}

Malgré son apparente simplicité, la notion de première fois est un élément essentiel des récits biographiques et historiographiques, du fait de sa récurrence et de la variété des événements pouvant être abordés par les locuteurs. Bien que l'expression soit souvent associée à un sens sexuel en français, nous en proposons ici une définition plus large : un événement marquant, n'ayant jamais eu lieu auparavant, à l'échelle individuelle ou collective. On remarque d'ores et déjà la dimension temporelle de ce concept, puisqu'il est mis en rapport avec le passé, pour souligner la différence avec un état de fait antérieur, et le futur, dans la mesure où une première fois peut marquer le début d'une série ou ne rester qu'une occurrence unique, comme le remarque Pomian lorsqu'il parle de «l'émergence d'une nouvelle figure qui se détache sur le fond du déjà-vu » pour définir le concept d'événement historique dans L'ordre du temps (1984:17). Quoi qu'il en soit, cela en fait un élément éminemment racontable, en particulier dans le corpus que nous avons choisi d'étudier : les documentaires musicaux.

Riches en récits biographiques et autobiographiques oraux, les documentaires que nous avons intégrés à notre corpus portent sur la musique populaire anglophone. En effet, ces films consacrés à des chanteurs (Bob Dylan, George Harrison, Joe Strummer Patti Smith, Leonard Cohen, Sixto Rodriguez), des groupes (les Rolling Stones, The Band, les Sex Pistols, LCD Soundsystem) ou des mouvements musicaux (la Britpop) sont constitués de nombreuses interviews d'artistes ou de témoins réunies dans 
une démarche explicitement historiographique: il s'agit de retracer la carrière d'un groupe ou d'un chanteur ou de rendre compte de l'esprit d'une époque. Les films intègrent donc des images d'archives et des interviews, réalisées plusieurs décennies plus tard, contenant des anecdotes et des récits de vie censés éclairer les épisodes marquants de l'histoire musicale des soixante dernières années. On peut donc parler d'une articulation entre un macro-récit filmique polyphonique et les micro-récits qui le composent. Cette articulation comporte une dimension thématique, par exemple la confrontation de différents points de vue sur un même événement, mais aussi chronologique, puisque le réalisateur cherche à rendre compte de la succession des événements narrés via le travail de montage et l'agencement des témoignages. La technique filmique sert ici un travail de "mise en intrigue ", c'est-à-dire "l'opération qui tire d'une simple succession une configuration. » (Ricœur, 1983: 127).

Les premières fois sont nombreuses dans ces films documentaires, puisque l'on dénombre 79 récits de première fois pour onze films et un corpus de 128000 mots environ. Nous en retiendrons trois grandes catégories : les rencontres (amicales, amoureuses ou professionnelles), les débuts artistiques (les événements retenus pouvant être de natures diverses selon les intervenants) et les nouveautés, qu'il s'agisse des découvertes (en général en tant qu'auditeur) ou des innovations (par les artistes). Cette dernière catégorie est la plus représentée (39 récits), car c'est justement celle qui est accessible au plus grand nombre de témoins, contrairement aux débuts musicaux (16 occurrences), les rencontres occupant une position médiane dans cette répartition (24 occurrences). Sur le plan syntaxique, les récits de première fois peuvent être signalés par le syntagme "first time » (22 occurrences), comme dans la citation de Johnny Rotten que nous avons mise en exergue et dans laquelle le modal would, la deuxième personne du singulier et la gradation inhabituelle de l'ordinal first («so first time») tirent la référence vers le type. On remarque que le syntagme first time est plus souvent présent en début d'anecdote, dans une phase d'annonce, bien qu'on le retrouve parfois en position de reprise et de conclusion. De manière générale les primo-récits, qui peuvent également être marqués lexicalement, par exemple par le verbe meet (rencontrer), l'adjectif new (nouveau) ou l'adverbe never (jamais), se trouvent plutôt au début des films, ce qui n'est pas surprenant car les narrations filmiques suivent une chronologie linéaire dans l'immense majorité des cas. Les débuts de carrière sont donc plus susceptibles de comporter des premières fois méritant d'être narrées.

Ce haut degré de racontabilité explique le nombre important de récits de première fois dans notre corpus: une nouveauté est un objet de narration potentielle fort, dans la mesure où un changement d'état du monde est 
susceptible de susciter l'intérêt. (Ochs et Capps, 2001 : 33). Néanmoins, l'attractivité narrative ne peut à elle seule expliquer cette récurrence, d'autant que les intervenants eux-mêmes commentent, implicitement ou explicitement, la prépondérance de ces épisodes à une autre échelle : celle du récit de soi. En effet, la définition que donne Delory-Monberger (2009: 24) du concept semble s'appliquer aux témoignages des chanteurs : «(...) une forme de l'expression individuelle, un objet social, produit d'une pratique codifiée répondant à une demande biographique institutionnalisée. » Du fait de leur importance quantitative et de leurs succès, on peut parler d'une "pratique institutionnalisée » dans le cas des biographies de stars, au sein desquelles la première fois occupe une place importante que nous essaierons de comprendre et définir.

Pour ce faire, nous nous appuyons sur le concept de récit des origines, à savoir la tendance à présenter un événement ancien comme la source, voire la prophétie, d'une carrière future. Cette tendance du regard rétrospectif à donner du sens à des événements du passé du fait de leur influence supposée sur l'avenir est un trait caractéristique des discours inclus dans les documentaires rock. Cela s'explique par la représentation qui est faite du parcours individuel, qui suit, comme le remarque Delory-Monberger, celle du récit de formation dans lequel le protagoniste agit sur son évolution au travers de ses choix et de ses actions (2009 : 33), et par le ressort discursif identifié par Ruth Amossy (1991) dans les biographies de stars hollywoodiennes : la confrontation de l'image publique de la star avec sa personnalité privée, supposée "authentique ». Nous verrons comment ces deux entités sont abordées et bien souvent réconciliées grâce aux récits des premières fois

Il ne faut pas oublier non plus que les récits de vie que nous nous proposons d'étudier sont pris dans un récit plus vaste, celui de l'histoire du rock. Or, comme le remarque Claude Chastagner (2011), la « culture rock » est éminemment consciente de sa propre historicité et s'avère friande de récits et de commémorations. Il va sans dire que les documentaires, associant musique et témoignages, sont des outils privilégiés pour écrire l'histoire du rock. On remarque également que cette histoire et cette culture revendiquées valorisent beaucoup les première fois, notamment les innovations et les créations qui sont perçues comme l'expression de l'individualité des musiciens (Chastagner, 2011 : 38). On voit donc en quoi les premières fois des biographies de stars résonnent dans l'histoire du rock, dans la mesure où les artistes ont contribué à écrire cette histoire grâce à leurs faits d'armes, racontés et repris par un public conquis par leur audace et la rébellion qu'ils étaient supposés incarner. On constate également qu'ils 
contribuent à la reconstruire et se la réapproprier en témoignant dans les films.

Nous faisons donc l'hypothèse que les premières fois sont en réalité intégrées à trois récits: le récit filmique composite, le récit de vie et l'histoire du rock. Points de passage entre ces récits, elles serviraient donc des visées communes, notamment la reconstruction d'une continuité entre personne privée, personnage public et icône culturelle, ce qui expliquerait leurs convergences formelles et thématiques. Conscient de la particularité du médium et du champ culturel dans lesquels apparaissent ces récits, nous entendons en proposer une analyse discursive à partir d'exemples significatifs.

\section{Micro et macro-récits : anecdotes et récit documentaire}

L'articulation entre les anecdotes et le récit filmique évoquée plus haut est un phénomène qu'il convient d'envisager de façon dynamique : le documentaire est composé d'anecdotes multiples mises en écho selon le propos avancé par le réalisateur, mais les anecdotes sont elles aussi produites et commentées par les différents intervenants en fonction du thème du film. En d'autres termes, les interventions des témoins sont orientées vers un propos supposé du film et les locuteurs prennent généralement soin de justifier leur intervention. Les traces discursives de cette démarche se retrouvent à travers ce que Labov (1972) a nommé l'évaluation, à savoir les moyens mis en œuvre à l'oral pour indiquer le but d'un récit, sa raison d'être, sa pertinence. Notons que Labov avait d'abord fait l'hypothèse d'une suspension du récit pour en souligner sa racontabilité, avant d'opter pour un modèle plus diffus dans Language in the Inner City. On remarque que dans le contexte du documentaire, les anecdotes sont généralement évaluées en vertu de ce qu'elles sont censées apporter au récit de carrière : un exemple significatif quant à l'attitude ou la personnalité d'un artiste, un événement exceptionnel ayant eu un impact important sur sa carrière, une anecdote méconnue, etc. Comme le remarque Ricœur dans Temps et Récit (1983 : 127), «l'événement doit être plus qu'une occurrence singulière. Il reçoit sa définition de sa contribution au développement de l'intrigue ", et il en va de même pour ces récits filmiques. Les récits de première fois ne font pas exception et, bien souvent, les témoins justifient l'intérêt de leur histoire par le fait même qu'ils narrent un événement alors inédit. Par exemple, dans No Direction Home, Bob Dylan raconte sa découverte de Woody Guthrie, grande figure du folk, chanteur engagé et influence majeure lors de la première partie de sa carrière. On pourrait s'attendre à ce que son anecdote soit peu commentée, car étant le sujet du 
documentaire, tout récit de Bob Dylan est potentiellement pertinent. C'est pourtant l'inverse qui se produit :

[1] Woody Guthrie, he had a particular sound. And besides that, he said something to go along with his sound. That was highly unusual to my ears. He was a radical, his songs had a radical slant. I thought, "Ooh," you know, like... "That's what I want to sing. I want to sing that." I couldn't believe that I'd never heard of this man. You could listen to his songs, and actually learn how to live.

Woody Guthrie, avait un son particulier. Et en plus, il avait quelque chose à dire pour aller avec ce son. C'était extrêmement inhabituel pour moi. Il était radical, et ses chansons avaient un côté radical. Je me suis dit «Oh», vous savez, «c'est ce que je veux chanter, je veux chanter ça.» Je n'arrivais pas à croire que je n'avais jamais entendu parler de cet homme. On pouvait apprendre à vivre en écoutant ses chansons.

Cette découverte des chansons de Woody Guthrie, présentée comme une sorte d'épiphanie, est abondamment évaluée par Bob Dylan. Il fait d'abord un commentaire sur le caractère inhabituel de ces chansons, soulignant ainsi l'aspect doublement inédit de cette première fois : il découvre non seulement un chanteur, mais aussi une nouvelle manière de composer. Bien souvent, une réaction intense face à une découverte est avancée comme un élément d'évaluation justifiant la pertinence du récit, cette spontanéité du ressenti serait la garantie d'une expérience exceptionnelle : on la retrouve dans la découverte des Rolling Stones à la radio par le jeune Joe Strummer (Joe Strummer) ou celle des Beatles à Hambourg par celui qui devint leur ami, Klaus Voormann (George Harrison). Du reste, une première impression correspondant à un jugement juste (par exemple un producteur remarquant le talent et le potentiel d'un jeune chanteur, comme Albert Hammond découvrant Bob Dylan) est tout aussi racontable qu'une impression fausse et corrigée par la suite. Ainsi, Patti Smith évoque dans Dream of Life sa réaction lorsqu'elle a rencontré l'acteur et réalisateur Sam Shepard, avec qui elle eut plus tard une liaison : «quel enfoiré de péquenaud! » [that's one hillibilly son of a bitch]

On constate également que dans un deuxième temps, Bob Dylan rapporte son désir d'imitation en citant ses pensées de l'époque, un moyen d'évaluation interne relevé par Labov (1972 : 372) consistant à rapporter son sentiment sur l'épisode sous la forme d'une réflexion intradiégétique plutôt que comme un commentaire a posteriori. De plus, on peut supposer qu'à travers cette pensée, Bob Dylan fait implicitement allusion aux conséquences qu'a eues cette découverte sur sa carrière de chanteur, puisqu'il a choisi d'imiter le style de Woody Guthrie (on peut d'ailleurs 
penser à sa chanson «Song To Woody Guthrie »). On aurait donc ici une deuxième forme d'évaluation, à savoir la référence aux conséquences de l'événement narré sur le protagoniste et le narrateur: la découverte de Woody Guthrie mérite d'être racontée parce que Bob Dylan s'en est inspiré par la suite.

Le récit des premières fois est donc un moyen privilégié d'intégration des témoins dans le film, ce que l'on remarque au fait que les stratégies d'évaluations avancées par les intervenants font souvent appel à deux caractéristiques importantes du concept : l'inattendu et l'ouverture sur les conséquences de l'épisode. Cette deuxième caractéristique est du reste exploitée par les réalisateurs dans la construction de leur récit, ce qui contribuerait à expliquer la récurrence des premières fois : les épisodes sont évoqués parce qu'ils permettent un séquençage dans la carrière du chanteur ou du groupe dont il est question. En d'autres termes, la première fois présentée comme un événement marquant permet de marquer des passages d'une période à une autre et donc de faire progresser le récit par étape, la discontinuité par rapport à l'état précédent étant d'ailleurs une caractéristique essentielle du concept d'événement soulignée par Pomian (1984: 33). Ainsi, certains épisodes se trouvent dotés d'une importance symbolique forte, exprimée soit par l'insistance explicite des témoins soit par des moyens filmiques (accumulation des témoignages sur un même événement, recours aux images d'archives, etc.). Il s'agit donc ici d'une articulation entre micro-récits et macro-récit puisque la structure même du macro-récit est dessinée grâce à un micro-récit significatif. Un cas intéressant de transition filmique autour d'un récit de première fois se trouve dans le film Live Forever, qui ne traite pas à proprement parler d'un groupe mais d'un mouvement musical : la Britpop, c'est-à-dire l'émergence de groupes britanniques extrêmement populaires au milieu des années 1990, notamment Blur et Oasis. Louise Wener, du groupe Sleepers, raconte un épisode qui, d'après elle, a marqué la fin du mouvement :

[2] We were recording our last album and Robbie [Williams] was recording his first in the studio next door. (...) And you sort of knew that this was gonna be the new force. Robbie Williams making music kind of like Oasis. So once he was doing it, you knew it was over, that was the moment that it was finished.

On enregistrait notre dernier album et Robbie enregistrait son premier dans le studio d'à côté. (...) Et on se doutait un peu que ça allait être la nouvelle force. Robbie Williams faisant de la musique qui ressemblait un peu à Oasis. Maintenant qu'il faisait ça, on savait que c'était terminé, qu'à ce moment-là c'était fini. 
Dans son commentaire de l'évènement, qui n'a du reste pas eu de grand retentissement dans l'histoire du rock, Louise Werner souligne le caractère transitionnel de l'anecdote: le dernier album de son groupe, assimilé au mouvement Britpop, correspond temporellement et spatialement au premier album de Robbie Williams. Ce premier album serait donc une forme d'adoubement, " la nouvelle force » prenant la place du groupe phare du moment, Oasis, qui venait de sortir un album décevant. Cette succession était donc un début autant qu'une fin, puisqu'elle marque l'essoufflement de la Britpop. Notons que le film propose d'autres passages symboliques pour marquer la fin de cette période d'euphorie culturelle, notamment la mort de Lady Diana, mais que pour cette intervenante la fin d'une période musicale ne peut être marquée que par l'ouverture sur une nouvelle ère, donc par une nouveauté, une première.

Les premières fois servent donc d'articulation discursive et chronologique au niveau du récit filmique : elles permettent aux témoins de s'insérer dans le macro-récit et de justifier la pertinence de leurs interventions et aux réalisateurs de proposer une chronologie séquencée et rythmée par des événements à haute teneur symbolique. Néanmoins, il ne faudrait pas y voir qu'un outil documentaire, puisque les premières fois jouent un rôle tout aussi central dans la construction des récits de soi, en particulier en ce qui concerne les débuts de carrière et les origines de rocks stars.

\section{Origines et métamorphose : (re)construction de la personne publique}

Dans Les idées reçues (1991), Ruth Amossy propose une étude des biographies des stars hollywoodiennes dont les ressorts narratifs et discursifs sont assez similaires à ceux des documentaires rock. Elle explique notamment que la parole de ces stars, présentée comme authentique puisque le genre l'exige, est confrontée à la vision stéréotypée du public : le masque de la star est assumé ou rejeté mais il ne peut être ignoré (1991 :143). Elle remarque également que la star, devenue narrateur à la première personne «donne forme à son être ", qu'elle procède à une " mise en scène du moi » (1991 :151). Or, le récit de soi est bien souvent celui d'une métamorphose, qui permet de ménager les deux faces de la star : la personnalité publique connue du plus grand nombre et la personnalité « réelle » qui aspire à faire entendre sa voix.

Le concept de métamorphose nous semble tout à fait pertinent pour étudier les documentaires musicaux en général et les premières fois en particulier. En effet, on remarque que les débuts artistiques constituent un passage incontournable dans les récits de vie et sont présentés comme la 
matrice d'où aurait émergé le chanteur ou le groupe. Cela permet de ménager une continuité et une rupture : la continuité est préservée puisque c'est le même jeune artiste qui s'est trouvé transformé par un épisode significatif, mais il y a bel et bien une rupture dans le passage de l'anonymat à la célébrité ou de celui d'auditeur admiratif à artiste créatif. Il est du reste intéressant de constater que les épisodes avancés pour marquer le début d'une carrière en tant qu'artiste sont forts divers, et peuvent relever d'une pratique musicale professionnelle ou en amateur: premier instrument, première répétition, premier concert, premier disque, etc. Un cas fort intéressant concerne les premières compositions, notamment parce qu'il illustre une évolution de l'industrie du disque quant à la perception des rôles relatifs des compositeurs et des interprètes : nombre de musiciens ayant émergé dans les années 60 (Bob Dylan, les Beatles, les Rolling Stones) ont commencé par sortir des reprises de standards comme cela se faisait depuis le milieu des années 50, mais tous décrivent leur première composition comme un passage marquant. Par exemple Keith Richards et Mick Jagger témoignant dans Crossfire Hurricane :

[3] Mick Jagger: We started experimenting writing, maybe we weren't good at it but we tried anyway. Not everybody can write.

Keith Richards: We went on to write a whole lot of crap. Months and months and months to write a song for the Stones, you know, it's a whole other trip.

Mick Jagger: The first song I remember writing with the Rolling Stones was "Tell me", which was a hit. It was really gratifying cause we had only done these cover songs.

Mick Jagger : On a commencé à essayer d'écrire, peut-être qu'on n'était pas très bons, mais on a essayé quand même. Tout le monde n'est pas capable d'écrire.

Keith Richards : On a écrit un paquet de conneries. Des mois et des mois pour écrire une chanson des Stones, vous savez, c'est un tout autre délire.

Mick Jagger : La première chanson que je me souviens avoir écrite pour les Rolling Stones, c'était «Tell Me», c'était un tube. C'était vraiment gratifiant parce qu'on n'avait fait que des reprises.

On remarque que le passage d'un groupe de reprises à un groupe qui chante ses propres chansons est valorisé, malgré la difficulté du processus et les résultats pour le moins décevants décrits par Keith Richards. Néanmoins, la hiérarchie est claire : le succès de «Tell Me » est "gratifiant » d'après Mick Jagger, et il est opposé aux reprises, jugées inférieures. Cette première fois est donc présentée comme une étape clé dans l'évolution des Rolling Stones, qui sont à la fois les mêmes (des rock stars débutantes) et différents (des compositeurs à succès, donc des artistes plus légitimes). 
La confrontation avec l'image publique est un élément structurant fort dans les documentaires, d'autant qu'il y est souvent fait référence pour avancer un discours contredoxique, qui cherche à contredire ce que l'histoire du rock a retenu de certains chanteurs. Pourtant, lorsqu'on analyse les éléments avancés pour décrire les débuts artistiques de chacune des figures dépeintes dans les documentaires, on remarque que les traits saillants de leur image publique y sont déjà présents. C'est donc en ce sens que nous parlons d'un récit des origines, quasi prophétique, puisque le destin des artistes semble tracé dès leurs premiers pas. Il va sans dire que le regard rétrospectif tient une place prépondérante dans ce phénomène, puisqu'il est tentant de relire le passé à la lumière des événements survenus depuis. Berstein (Foregone Conclusions, 1994), cité par Ochs et Capps (2001 : 5), parle à ce propos de «backshadowing » à savoir la tendance qu'ont les narrateurs à juger les protagonistes de leur récit en vertu de l'issue qu'ont finalement connue des événements passés, "comme si ces derniers avaient pu la connaître ». Il n'empêche que cette métamorphose originelle, cette première fois majuscule, permet de rejouer la construction de l'image publique de ces chanteurs. Prenons quelques exemples :

[4] Theodore: And then you hear this voice. Strange voice.

Coffey: Finally, we walked through the smoke, and I looked, and there in the far corner I saw... I could see the shadow of a man and I couldn't see his face. I said, "What's the deal?" So we got a little closer...

Theodore: And you see this guy with his back to you. So all you see is his back and he's in a corner, singing. It was an ethereal scene, if you will. Foggy night, foghorns, smoke so thick you couldn't see through it. And here's this voice.

Theodore: Et là, on entend cette voix. Une voix étrange.

Coffey : On a fini par traverser la fumée, et j'ai regardé, et dans le coin opposé j'ai vu... Je distinguais la silhouette d'un homme mais je ne voyais pas son visage. J'ai dit: «Qu'est-ce que c'est cette histoire ? » Alors on s'est approchés.

Theodore : Et on voit ce type de dos. On ne voit que son dos, il chante dans un coin. C'était une scène éthérée si vous voulez. Une nuit de brouillard, des cornes de brume, une fumée si épaisse qu'on n'y voyait rien. Et puis cette voix.

Mike Theodore et Dennis Coffey, Searching for Sugar Man

[5] The first time I actually wrote anything that I thought had a significance was after my father died when I was nine. (...) The first poetry that ever affected me was in the synagogue, in the liturgy and the Bible stories. And that would send shivers down my spine. (...) I 
started writing poetry to girls. Tried to get girls interested in my mind.

La première fois que j'ai écrit quelque chose qui comptait, c'était après la mort de mon père, quand $j$ 'avais neuf ans. (...) La première fois que j'ai été touché par la poésie, c'était à la synagogue : la liturgie et les récits bibliques. Ça me donnait des frissons dans le dos. (...) J'ai commencé à écrire des poèmes pour les filles. Pour tenter de les intéresser à mon esprit.

Leonard Cohen, I'm your man

[6] All our first rehearsals were a nightmare. I couldn't hold a damn note, Paul couldn't really keep time, I couldn't play guitar. I fucking hated it. It was just a fucking noise, but I just stuck in there, because that's all I had.

Toutes nos premières répétitions ont été un cauchemar. Je ne pouvais pas tenir une foutue note, Paul n'arrivait pas à garder la mesure, je n'arrivais pas à jouer de la guitare. Je détestais ça putain. C'était qu'un putain de bruit, mais je suis resté, parce que c'était tout ce que j'avais.

Johnny Rotten, The Filth and the Fury

[7] We needed a good guitar player. Both John and I played a bit of guitar, but we couldn't really solo.

We weren't that good. And I said, "I know this guy. He's a bit young, but he's good." John said, "Well," you know," let's meet him". So I said to George, "You want to meet these guys I'm in a group with?" "Yeah." So he brought his guitar. We were all on the top deck of a double-decker bus in Liverpool, round where John lived, a place called Woolton, and nobody was on the bus. It was late at night. And John said, "Well, go on then. Let's see you play" to George. I said, "Go on, go on, get your guitar out." So George unpacked his guitar, got it out, and he played the thing called Raunchy, which is...

Il nous fallait un bon guitariste. John et moi savions jouer un peu, mais on n'était pas capables de faire de solo. On n'était pas assez bons. Alors j'ai dit : "Je connais un type. Il est un peu jeune mais il est bon. "John a dit : "Bon, d'accord, amène-le. " Alors j'ai dit à George: "Tu veux rencontrer les mecs de mon groupe?" "Ouais. " Alors il a apporté sa guitare. Nous étions à l'étage d'un bus à Liverpool, près de chez John, un endroit qui s'appelait Woolton, et le bus était vide. Il était tard. Alors John a dit à George: "Bon, vas-y. Voyons comment tu joues. " J'ai dit : "Vas-y, vas-y, sors ta guitare. " Alors George a déballé sa guitare, il l'a sortie et a joué un air qui s'appelle Raunchy et qui donne...

Paul McCartney, George Harrison: Living in the Material World 
Ces premières fois, fort diverses (la découverte de Sixto Rodriguez par ses producteurs, les premiers écrits de Leonard Cohen, les premières répétitions des Sex Pistols, et l'audition de George Harrison par John Lennon), sont pourtant conformes à l'image stéréotypée de ces artistes. De plus, on remarque qu'ils partagent cette image avec d'autres musiciens présents dans notre corpus, le plus souvent en fonction de leur genre musical de prédilection ou en fonction de l'axe narratif du film. Ainsi, Sixto Rodriguez, sujet de Searching for Sugar Man, est présenté comme une voix sans visage et tout le récit est tourné vers la recherche de ce chanteur mystérieux par le réalisateur. Dans cette perspective, la découverte aveugle par ses producteurs est conforme à l'image véhiculée dans le film (on remarque que, de la même manière dans Shut Up and Play the Hits, documentaire sur la fin du groupe LCD Soundsystem, le récit de débuts «accidentels » est justement mis en perspective avec la fin programmée du groupe). Leonard Cohen, connu pour la profondeur de ses textes, ses poèmes et ses romans, mais aussi pour sa réputation d'homme à femmes et sa ferveur religieuse (notamment du fait de sa conversion au bouddhisme) propose toutes ces pistes au moment de narrer ses premiers pas de créateur. Il est intéressant de remarquer que d'autres chanteurs à textes et artistes éclectiques reconnus en font de même, notamment Bob Dylan qui explique que ses premières sérénades ont fait ressortir «le poète en lui » et Patti Smith qui a commencé par écrire des poèmes qu'elle a mis en musique par la suite. L'idéologie punk est présente dans le récit de Johnny Rotten : des lacunes techniques compensées par l'énergie, une musique assimilée à du bruit, une forme de nihilisme et de rébellion face à une situation sociale défavorisée. On retrouve ces topoï dans les documentaires sur Joe Strummer qui a commencé par le ukulélé en croyant qu'il serait plus simple d'apprendre à jouer d'un instrument à quatre cordes et qui garde un souvenir ému des premiers pas laborieux des Clash. Enfin, les Beatles semblent avoir déjà trouvé leur place dans ce bus de Woolton: Lennon et McCartney comme duo moteur du groupe, George Harrison en musicien mutique et effacé (Paul McCartney rappelle qu'il était plus jeune et ne lui donne aucune réplique au discours direct pendant la scène du bus). Notons que Ringo Starr a rejoint le groupe plus tard et que les autres membres de ce premier groupe restent dans l'ombre. La situation la plus comparable est, sans surprise, celle des Rolling Stones, dont le récit de carrière dans Crossfire Hurricane tourne principalement autour des rapports entre Keith Richards et Mick Jagger.

Si le récit des débuts artistiques, ainsi que celui d'autres premières fois significatives, permettent de rejouer la construction de l'image publique de la rock star, ceux-ci sont également les étapes d'une carrière reconfigurée 
comme un récit de formation. Ainsi que l'explique Delory-Monberger, ce cadre narratif et cognitif a une influence forte sur la «condition biographique» moderne, puisqu'il suppose la perfectibilité de l'être humain, capable d'apprendre de ses expériences et de ses rencontres et d'être le moteur de son développement (2009 : 67). Le récit de carrière est donc présenté comme celui d'une auto-réalisation, celle à laquelle l'individu moderne est tenu, puisque ses réussites et ses échecs lui seraient directement imputables (2009: 21). Certes, les choix et les actions présentés comme ayant eu une influence sur le cours d'une carrière ne se limitent pas aux premières fois, mais celles-ci sont tout de même mises en avant, notamment lors des débuts des artistes, période où la possibilité de poursuivre est forcément plus fragile. On souligne et on valorise l'audace et l'originalité de certains choix, ceux que la rock star a su faire et qui la distinguent de son public, resté dans l'anonymat. Un topos, que l'on retrouve dans le récit de formation, est celui de la rupture spatiale : faire le choix de quitter sa ville ou son pays pour glaner le succès ailleurs (notamment aux Etats-Unis dans le cas des groupes britanniques comme les Beatles, les Rolling Stones ou les Clash) est présenté comme une décision difficile, risquée, mais payante. C'est le cas de Levon Helm, batteur de The Band, qui évoque New York dans The Last Waltz :

[8] A dream come true. Fascinating. Scary. Kind of hard to take the first time. You have to go there about two or three times before you can fall in love with it. But that happens eventually. (...) Yeah, New York, it was an adult portion. It was an adult dose. So it took a couple of trips to get into it. You just go in the first time and you get your ass kicked and you take off. As soon as it heals up, you come back and you try it again. Eventually, you fall right in love with it.

Un rêve devenu réalité. Fascinant. Effrayant. Assez dur à encaisser la première fois. Il faut y aller deux ou trois fois avant de pouvoir tomber amoureux de la ville. Mais ça finit par arriver. (...) Ouais, New York, c'était la taille adulte. Une part d'adulte. Il a fallu un ou deux voyages pour s'y faire. Tu y vas la première fois, tu te fais botter le cul et tu t'en vas. Mais dès que ça guérit, tu y retournes et tu réessayes. Finalement, tu tombes amoureux.

On devine dans cet exemple l'importance de New York dans la géographie symbolique des musiciens rock, mais on voit également que la route vers le succès de The Band, groupe originaire du Canada, est présentée comme le fruit d'une décision et d'une forme de persévérance face à un premier échec, le déplacement spatial se doublant d'un passage à l'âge adulte dans le récit du musicien. 
On constate donc que les premières fois sont mises en avant pour retracer les métamorphoses successives qui ont fait la rock star, elles servent de points de passage entre le passé et l'avenir d'une part, et d'autre part entre la personne « réelle » que la démarche documentaire doit permettre de faire émerger (comme le remarque Cohen, 2012) et la personnalité publique qui suscite l'intérêt des fans. On remarque également un certain conformisme discursif, notamment dans la reconstruction des carrières qui suivent le modèle du récit de formation, rythmé par les choix et les décisions du protagoniste. Mais il serait malvenu de ne pas tenir compte de la spécificité de la communauté de pratique dans laquelle ces discours émergent, celle des rockers, et de ne pas souligner l'influence de la culture rock sur ces récits de soi.

\section{L'Histoire du rock : un métarécit structurant}

Nous empruntons le concept de «métarécit » à Delory-Monberger qui le définit ainsi (2009 : 98) : ces récits «transposent au plan de la durée et de l'histoire individuelle les caractéristiques structurelles et le système d'actants et de valeurs des grands récits dont ils sont l'émanation. » Elle remarque d'ailleurs que ces métarécits «peuvent étendre leur influence sur des aires plus ou moins vastes, concerner des domaines plus ou moins larges de l'existence, depuis les récits génériques porteurs des représentations collectives de tout un espace culturel et sociétal («le monde occidental») jusqu'aux biographies typiques spécifiant des trajectoires dans tel ou tel secteur de la vie sociale et professionnelle (le cursus de l'élève ou la carrière du manager) (...) » Nous faisons donc l'hypothèse que l'histoire du rock, étendue à la fois au milieu professionnel qu'est l'industrie de la musique et à une communauté de pratique culturelle constituée par le public, constitue un métarécit dans lequel le récit de vie de artistes vient s'inscrire.

Notons, que là encore il ne s'agit pas d'un phénomène unilatéral, d'une matrice narrative d'où seraient issus des récits individuels, puisque l'incarnation de la culture rock (phénomène complexe, composite et bien souvent contradictoire, brillamment analysé par Claude Chastagner dans son étude éponyme) prend également forme grâce au récit de vie de rock stars. Ainsi, être rock serait synonyme de vivre rock, et le terreau de ce phénomène culturel, d'après Chastagner, ne serait autre que l'identification du public, d'où l'importance des icônes culturelles aux biographies maintes fois ressassées. Il propose d'ailleurs une vision assez critique de ce phénomène, qu'il décrit ainsi : «l'esprit rock est avant tout fait d'identification à des figures tutélaires dont on retiendra quelques gestes marquants, quelques épisodes significatifs, à partir desquels il sera possible d'imaginer une existence. Peu importe l'exactitude de la biographie ainsi 
fabriquée. (...) De tout ce qui s'écrit sur le rock, la forme la plus fréquente, la plus populaire, c'est la biographie, là où la précision documentaire côtoie le romanesque, où la fiction la plus échevelée est parfois en deçà de la réalité, où la démesure est de rigueur. » (2011 : 91-92)

Contrairement à ce que pourrait laisser croire la place prépondérante accordée dans la culture rock à la spontanéité et à une forme de plaisir prélangagier, celle-ci serait donc avant tout une construction discursive, narrative et historiographique tournée vers des événements jugés dignes d'être retenus et transmis. Dans cette perspective, les premières fois prennent toute leur importance, d'autant qu'elles cristallisent une dialectique à l'œuvre dans l'histoire du rock : l'intégration à une tradition et l'affirmation d'une originalité novatrice.

On remarque que nous avons pu apercevoir des traces de ces dynamiques dans certains exemples déjà cités, notamment l'hommage de Bob Dylan à Woody Guthrie et la valorisation de la composition par les Rolling Stones. L'intégration à une lignée artistique est un phénomène extrêmement fréquent dans les documentaires musicaux; elle passe généralement par une rencontre avec un artiste ou par une émotion d'auditeur (en concert ou via un enregistrement). Un exemple frappant de cette affirmation d'une lignée se trouve dans le film Joe Strummer, ce qui pourrait paraître surprenant dans la mesure où le mouvement punk, dont les Clash ont été les pionniers, cherchait justement à rompre avec les standards du passé. Néanmoins, Joe Strummer n'a jamais caché qu'il avait été inspiré par d'autres groupes, et il a par la suite servi de modèle à de nombreux musiciens, parmi lesquels Bono (U2) :

[9] Joe Strummer: That's when the Pistols came in and blew everything away. (...). They came out of nowhere and they came to support us one night at the National House. It was electrifying. They were like a million years ahead. (...)

Steve Jones (Sex Pistols): I imagine he must have seen us play and figured the rockabilly wasn't working and this was the way to go.

Joe Strummer: Et là les Sex Pistols ont débarqué et ils ont fait tout exploser (...). Ils sont arrivés de nulle part pour être notre première partie un soir au National House. C'était électrisant. Ils avaient un million d'années d'avance. (...)

Steve Jones (Sex Pistols): J'imagine qu'il a dû nous voir jouer et se dire que le rockabilly ne marchait pas et que c'était la voie à suivre.

[10] Bono: It's all about The Clash being your first ever rock show. I was 17 years old, hanging out with my mates and we got into Trinity College, Dublin. (...) I was terrified, I was excited, and rock $n$ roll was not entertainment in that moment, it was a matter of life and death. Something much more serious. So me and my mates stayed 
up, trying to figure out how we could be in bands that meant as much as The Clash.

Bono : C'est ça le truc, d'avoir vu les Clash pour son premier concert. J'avais 17 ans, je traînais avec mes potes et on est allés à Trinity College, à Dublin. (...) J'étais terrifié, j'étais excité, et le rock $\mathrm{n}$ roll n'était plus un divertissement à ce moment-là, c'était une question de vie ou de mort. Quelque chose de beaucoup plus sérieux. Alors mes potes et moi, on est restés debout pour essayer de trouver comment on pouvait être dans des groupes qui comptaient autant que les Clash.

Même si les ressemblances musicales ne sont pas forcément évidentes, le film propose, grâce à ces récits de premières fois, une lignée Sex PistolsThe Clash-U2, puisque la formation et l'évolution de chacun de ces groupes trouvent leurs racines dans la découverte d'un autre groupe. Il faut du reste souligner que les documentaires offrent généralement une image positive, voire élogieuse, des chanteurs dont il est question, ce genre de compliments et d'affirmations sont donc de bon ton dans ce contexte. Ainsi Nick Cave parle de son admiration pour Leonard Cohen, Patti Smith cite Bob Dylan, George Harrison Elvis Presley, etc.

L'originalité novatrice et la rupture avec la tradition constituent l'autre force à l'œuvre dans l'historiographie du rock, ce que l'on peut expliquer par des stratégies commerciales (l'attrait de la nouveauté) et par une tendance générale de l'histoire des arts à valoriser les innovations et les avancées techniques. De grands épisodes sont retenus et ressassés- les premiers concerts des Sex Pistols en font d'ailleurs partie- et les chanteurs et autres témoins, qui les ont vécus du côté du public, sont naturellement interrogés dessus. Les premières fois sont donc racontées car elles illustrent la figure de l'artiste créateur, du pionnier et, d'une certaine manière, du rebelle prêt à aller à l'encontre de la tradition. Un des exemples les plus frappants est bien sûr celui de Bob Dylan, dont le passage du folk au rock ne s'est pas fait sans heurts, ce que raconte en substance No Direction Home, puisque le film s'arrête d'ailleurs après sa tournée houleuse en GrandeBretagne en 1966. Sa connaissance de la tradition folk et sa conscience politique sont évoquées à plusieurs reprises dans la première partie du film, comme pour mieux montrer avec quelle force le chanteur, déjà novateur dans ce genre, a su s'en détacher. De plus, cette rupture, avec la tradition et avec une partie de son public, se double dans le récit de vie d'une rupture avec sa petite amie de l'époque : Joan Baez, égérie du folk et de la chanson contestataire. Cela n'est pas anodin, car ce brouillage des frontières entre vie publique et vie privée est justement au cœur de la construction du récit rock, via la biographie de ces icônes. 
[11] So even if he never considered himself a protest singer, he was a protest singer. And he saw what was happening all around him and everybody else saw what was happening all around them but he voiced that for the first time.

Donc même s'il ne s'est jamais vu comme un chanteur engagé, c'était un chanteur engagé. Et il voyait tout ce qui se passait autour de lui et tout le monde voyait ce qui se passait autour d'eux, mais il a été le premier à l'exprimer.

Joan Baez, No Direction Home

[12] "Like a Rolling Stone" definitely broke through somewhere. I didn't feel like radio had ever played a song like that before. I know I'd never heard a song like that before.

«Like a Rolling Stone » était une réelle avancée. Je n'avais pas l'impression qu'une telle chanson était déjà passée à la radio. Je sais que je n'avais jamais entendu une chanson pareille auparavant.

Bob Dylan, No Direction Home

Il semblerait qu'il y ait donc bien une transposition des structures de l'histoire du rock dans les récits de vie des chanteurs, notamment en ce qui concerne la dialectique entre tradition et innovation et le brouillage des frontières entre vie privée et vie publique. Les premières fois jouent un rôle particulier dans ce système de correspondances, puisqu'elles permettent de souligner le caractère exceptionnel d'un événement qui a marqué à simultanément la vie de l'icône et l'histoire du mouvement culturel dont elle est l'incarnation.

\section{Conclusion}

Nous avons donc vu en quoi les premières fois constituent des points de passage au sein des divers récits auxquels elles sont intégrées, du fait de leur haut degré de racontabilité et parce qu'elles permettent d'établir une continuité entre la voix du narrateur retraçant sa propre vie, la personnalité publique reconstruite au fil de récit filmique composite et l'icône culturelle incarnant l'histoire du rock. Si le caractère exceptionnel, réel ou revendiqué, des rock stars se déploie dans ce corpus, il nous semble cependant que l'intérêt pour les premières fois dans le récit de soi dépasse le simple cadre de la musique populaire et démontre la prépondérance de ce récit des origines dans la démarche biographique. 


\section{Filmographie}

Crossfire Hurricane, Brett MoRGEN, Milkwood Films, 111 min, 2012.

George Harrison: Living in the Material World, Martin SCORSESE, Grove Street Pictures, 205 min, 2011.

Joe Strummer: The Future Is Unwritten, Julian TEMPLE, Nitrate Strummer/Parallel Films, 120 min, 2006.

Leonard Cohen: I'm Your Man, Liam Lunson, Lions Gate Films, 99 min, 2005.

Live Forever: The Rise and Fall of Brit Pop, John Dower, Passion Pictures, 86 min, 2002.

No Direction Home: Bob Dylan, Martin ScORSESE, Spitfire Pictures, 208 min, 2005.

Patti Smith: Dream of Life, Steven SEBRING, Thirteen/Clean Socks, 109 min, 2007.

The Filth and the Fury, Julian TEMPLE, Film Four, 108 min, 2000.

The Last Waltz, Martin ScORSESE, The Last Waltz Production, 112 min, 1978.

Searching For Sugar Man, Malik Bendjelloul, Red Box Films, 86 min, 2012.

Shut Up and Play the Hits, Will Lovelace \& William Southern, Pulse Films, 108 $\min , 2012$.

\section{Bibliographie}

AMOSSY R., 1991, Les idées reçues : Sémiologie du stéréotype, Paris, Nathan.

CHASTAGNER Cl., 2001, De la culture rock, Paris, PUF.

COHEN Th. F., 2012, Playing to the Camera: Musicians and Musical Performance in Documentary Cinema, Londres, Wallflower.

DELORY-MOMBERGER Ch., 2010, La condition biographique, Essais sur le récit de soi dans la modernité avancée, Paris, Téraèdre.

LABOV W., 1972, Language in the Inner City, Philadelphia, University of Pennsylvania Press.

OCHS E. \& CAPPS L., 2001, Living Narrative, Cambridge (Massachusetts), Harvard University Press

POMIAN K., 1984, L'ordre du temps, Paris, Gallimard.

RICEUR Paul, 1983, Temps et récit (t.1) : L'intrigue et le récit historique, Paris, Seuil

Résumé :

Cette contribution cherche à évaluer le rôle joué par les récits de premières fois dans les récits de vie présents dans les documentaires consacrés aux grandes figures du rock. Les événements narrés sont de nature fort diverses mais partagent un certain nombre de traits communs sur le plan discursif : justification de la pertinence du témoignage, séquençage du macro-récit documentaire, possibilité de rejouer la métamorphose de la rock star et d'inscrire la biographie des artistes dans le métarécit que constitue l'histoire du rock.

\section{Mots-clés :}


Rock, documentaire, micro-récit, évaluation, métarécit

Abstract:

This paper aims at investigating the role of firsts in biographic narratives found in rock documentaries. Though interviewees may choose to tell very different events, the stories of these first times share a number of discursive characteristics: justifying the relevance of an anecdote, sequencing the documentary, replaying the metamorphosis of a rock star and inscribing the biographies of artists in the meta-narrative of rock history.

Keywords:

Rock music, documentaries, micro-narrative, evaluation, meta-narrative 